Revista de Psicología y Educación / Journal of Psychology and Education, 2020, 15(1), 77-86 (www.rpye.es) Doi: https://doi.org/10.23923/rpye2020.01.187

ISSN: $1699-9517$

\title{
Engagement académico e inteligencia emocional en estudiantes de Ciencias de la Salud
}

\author{
María del Carmen Pérez-Fuentes ${ }^{1 *}$, María del Mar Molero-Jurado², María del Mar Simón- \\ Márquez², Ana Belén Barragán-Martín², África Martos-Martínez², \\ Nieves Fátima Ruiz-Oropesa² y José Jesús Gázquez-Linares ${ }^{3}$
}

\author{
'Universidad de Almería / Universidad Politécnica y Artística del Paraguay \\ ${ }^{2}$ Universidad de Almería \\ ${ }^{3}$ Universidad Autónoma de Chile
}

\begin{abstract}
Resumen: El papel que juegan las emociones en los entornos académicos se encuentra estrechamente relacionado con el bienestar y compromiso de los estudiantes. Es por ello, que esta investigación tuvo como objetivo analizar la relación entre el engagement académico y los componentes de la inteligencia emocional en estudiantes universitarios y de Formación Profesional del ámbito de las Ciencias de la Salud en España. Para ello, se empleó una muestra de 263 estudiantes con una media de edad de 32,51 años. Se aplicó el Brief Emotional Intelligence Inventory for Senior Citizens y el Utrecht Work Engagement Scale. Este trabajo mostró que las mujeres afrontan con más fuerza sus estudios y presentan una mejor comprensión de sus emociones y sentimientos que los hombres. Además, no se encontraron diferencias entre ambos grupos para las titulaciones. Estos resultados tienen implicaciones prácticas acerca de la importancia de la inteligencia emocional en el desarrollo de intervenciones para la mejora del compromiso académico estudiantes de Ciencias de la Salud.
\end{abstract}

Palabras clave: Engagement académico, Inteligencia emocional, Estudiantes, Salud.

\section{Academic Engagement and Emotional Intelligence in Health Sciences Students}

\begin{abstract}
The role that emotions play in academic environments is closely related to the well-being and commitment of students. That is why this research aimed to analyze the relationship between academic engagement and the components of emotional intelligence in university students and Vocational Training in the field of Health Science in Spain. Forthis, a sample of 263 students with an averageage of 32.51 years was used. The Brief Emotional Intelligence Inventory for Senior Citizens and the Utrecht Work Engagement Scale were applied. This work showed that women face their studies more strongly and present a better understanding of their emotions and feelings than men. In addition, no differences were found between the two groups for the degrees. These results have practical implications about the importance of emotional intelligence in the development of interventions for the improvement of academic commitment students of Health Sciences.

Keywords: Academic Engagement, Emotional Intelligence, Students, Health.
\end{abstract}

Uno de los principales objetivos de los servicios sanitarios es optimizar la calidad de su atención, para ello el bienestar de los profesionales de este servicio tiene una gran implicación (Wersebe, Lieb, Meyer, Hofer, y

Recibido: 19/07/2019 - Aceptado: 24/09/2019 - Avance online: 25/1 1/2019 *Correspondencia: María del Carmen Pérez-Fuentes.

Universidad de Almería / Universidad Politécnica y Artística del Paraguay

C.P: 04120 , Almería, España.

E-mail:mpf421@ual.es

Pérez-Fuentes, M. C., Molero-Jurado, M. M., Simón-Márquez, M. M., Barragán-Martín, A. B. Martos-Martínez, A., Ruíz-Oropesa, N. F. y Gázquez-Linares, J.J. (Avance Online). Engagemen académico e inteligencia emocional en estudiantes de Ciencias de la Salud. Revista de Psicología y Educación, 15(1), 77-86, https://doi.org/10.23923/rpye2020.01.187.
Gloster, 2018). Este colectivo es uno de los más vulnerables debido a la interacción con los usuarios (Collet et al., 2018), y se ve afectado por distintos factores de riesgo y protección que pueden dar lugar al desarrollo dela aparición del burnout (Pérez-Fuentes, Molero, Gázquez, y Simón, 2019).Junto al síndrome de burnout, en el contexto laboral, una de las variables más estudiadas en los últimos años es el engagement. Desde la psicología 
positiva, ha sido definido como un estado psicológicode bienestar vinculado al trabajo y compuesto por tres dimensiones: vigor, dedicación y absorción (Schaufeli, Salanova, González-Romá, y Bakker, 2002). El vigor hace referencia a la dedicación de tiempo y esfuerzo de las tareas laborales, por su parte, la dedicación es la búsqueda significativa de objetivos caracterizados por la importancia, inspiración y orgullo, y la absorción, responde a la concentración completa en alguna tarea o actividad (Bakker, Albrecht, y Leiter, 2011).

Dentro de la psicología positiva,cuando hacemos referencia al engagement en el ámbito educativo, estamos hablando del engagement académico que alude a un estado debienestarpsicológico y compromiso hacialos estudios (Salanova, Bresó, y Schaufeli, 2005). Este constructo,ocasiona efectos positivos en las distintas variables relacionadas con el ámbito académico, como puede serla mejora del rendimiento académico (Barnett, Melugin, y Hernández, 2018; Casuso-Holgado et al., 2013). Un buen rendimiento académico, junto con el bienestar emocional, depende en gran medida del ajuste emocional comportamental de los estudiantes, encontrando así un peor rendimiento académico en los adolescentes que presentan una mayor dificultad en el ajuste emocional y comportamental (SánchezGarcía, Lucas-Molina, Fonseca-Pedrero, Pérez-Albéniz, y Paino, 2018).

El engagement es uno de los mecanismos que actúa como mediador entre la relación que se establece entre distintas variables (Zhang, Qin, y Ren, 2018), entre estas destaca, la adaptabilidad profesional y autoregulación (Merino-Tejedor, Hontangas, y Boada-Grau, 2016; Nilforooshan y Salimi, 2016), la autoeficacia (Lisbona, Palaci, Salanova, y Frese, 2018), la inteligencia emocional (Merino, Hontangas, y Petrides, 2018), entre otras. En este sentido, distintas investigaciones han encontrado que el engagement y la inteligencia emocional en estudiantes están estrechamente ligados (Maguire, Egan, Hyland, y Maguire, 2017; Perera y DiGiacomo, 2015; Serrano y Andrea, 2016). Pues, los factores relacionados con el engagement son tanto elementos laborales como personales, destacando entre estos últimos la autoeficacia y el optimismo, todos ellos, vinculados a la inteligencia emocional (Oriol-Granado, Mendoza-Lira, CovarrubiasApablaza, y Molina-López, 2017). Así, con el presente trabajo se pretende examinar la relación entre el engagement y las dimensiones de la inteligencia emocional en una muestra de estudiantes de Ciencias de la Salud.

De esta manera, el papel que juegan las emociones en los entornos académicos, es de especial interés puesto que la habilidad en el manejo de las emociones es uno de los predictores del bienestar (Carvalho, Guerrero, y Chambel, 2018) y del estrés, siendo los individuos con una mayor capacidad deadministrar las emociones los que afrontan mejor las situaciones de estrés (Urquijo, Extremera, y Villa, 2016). Precisamente, si los individuos no regulan correctamente las emociones, la capacidad de manejar las estrategias de afrontamiento activo se ve reducida (Morales, 2017).Por tanto, la inteligencia emocional actuaría como un factor protector y mediador ante dichas situaciones, lo cual podría explicar la relación positiva entre el engagement académico y la inteligencia emocional (Oriol-Granado et al., 2017; Oriol, Amutio, Mendoza, Da Costa, y Miranda, 2016). Pues, la investigación centrada en ambos constructos muestra como, en un estudio realizado con estudiantes, la inteligencia emocional predice el engagement académico (Durán, Extremera, Rey, Fernández-Berrocal, y Montalbán, 2006).Y de este modo, Merino-Tejedor et al. (2018) en su trabajo manifestaron que los universitarios con altos niveles de inteligencia emocional presentaban mayor engagement académico, estando esta relación mediada por la adaptabilidad profesional.

En comparación, Wach, Ruffing, Brünken, y Spinath (2016) indicaron que los universitarios que presentan una mayor capacidad ante las situaciones adversas, manifiestan mayor sensación de bienestar en el contexto educativo.Y si atendemos al sexo, las mujeres suelen experimentar mayor compromiso académico respecto a los hombres, pues suelen estar más involucradas en los 
estudios superiores, aunque esto les provoque agotamiento con más frecuencia que a los hombres (Salmela-Aro y Read, 2017).La edad es otra de las variables sociodemográficas que está vinculada al engagement académico. Así, los estudiantes universitarios más jóvenes suelen mostrar una mayor dedicación hacia los estudios, que los alumnos de más edad, por lo que la relación entre la edad y la dedicación suele ser negativa(Manzano, 2002).

Desde otra perspectiva, Martos et al. (2018) señalaron, en una muestra de estudiantes de Ciencias de la Salud, altos niveles de concentración académica, y una relación entre el engagement académico, el rendimiento académico y la percepción de autoeficacia. Además, se encontraron diferencias significativas según la titulación, concretamente en la variable intrapersonal de la inteligencia emocional. En cambio, en el trabajo de Serrano y Andrea (2016), se analizó la relación entre los componentes del engagement y las dimensiones de la inteligencia emocional, hallando una asociación entre vigor y dedicación, y la atención y reparación emocional, en una muestra de adolescentes.

Por otra parte, en un trabajo realizado con docentes universitarios, los autores destacaron que cuanto más alta era la claridad de sentimientos, reparación del estado emocional y la atención a las emociones, mayor era la dedicación, la absorción y el vigor (Andrade, Nobrega, y Franco, 2016).Pérez-Fuentes, Molero, Gázquez, y Oropesa (2018),en una muestra de enfermeros, hallaron que las mujeres obtenían altos niveles en el factor interpersonal e intrapersonal de la inteligencia emocional. Otras investigaciones, han encontrado puntuaciones altas en el grupo de hombres en las dimensiones manejo del estrés, adaptabilidad, y estado de ánimo (Azimi, AsgharNejad, Kharazi, y Khoei, 2010).

Una vez revisada la literatura científica sobre la relación que se establece entre el engagement y las diferentes dimensiones de la inteligencia emocional, encontramos un amplio volumen de trabajos de investigación, pero no tantos en poblaciones más específicas como los estudiantes de Ciencias de la Salud.

Teniendo en cuenta que el engagement está vinculado a niveles altos de inteligencia emocional. Se ha realizado este trabajo con el objetivo de analizar la relación entre el engagement académico y los componentes de la inteligencia emocional según el sexo, la edad y titulación, en una muestra de estudiantes de Ciencias de la Salud.

Partiendo de las investigaciones previas, nos planteamos las siguientes hipótesis: (1) existen diferencias en engagement académicoe inteligencia emocional según el sexo, siendo las mujeres las que puntuaran más alto en estado de ánimo e intrapersonal, y mostrarán mayor vigor para los estudios que los hombres; (2) existen diferencias en los componentes del engagement académico y las dimensiones de la inteligencia emocional en función de la titulación, siendo las puntuaciones mayores en los universitarios que en los estudiantes de Formación Profesional; (3) existe una relación negativa entre la edad y el factor dedicación del engagement; (4) existe una relación positiva entre las dimensiones de ambas variables.

\section{MÉTODO}

\section{PARTICIPANTES}

La muestra, obtenida a través de un muestreo intencional, estuvo formada por un total de 263 estudiantes de Ciencias de la Salud de nacionalidad española con un media de edad de 32.51 años $(D T=9.29)$. El 28.1\% $(n=74)$ eran hombres con una edad media de 32.05 $(D T=8.64)$ y el $71.9 \%(n=189)$ mujeres cuya edad media fue de 32.69 (DT=9.53).

En relación al tipo de estudios que cursaban, el $32.7 \% \quad(n=86)$ realizaban estudios universitarios de Ciencias de la Salud (Enfermería, Medicina, Psicología, Fisioterapia, Farmacia y Odontología) y el $65.8 \%(n=173)$ cursaban Formación Profesional (Técnico en Cuidados Auxiliares de Enfermería, Técnico en Anatomía Patológica, Técnico en Farmacia y Parafarmacia, Técnico de Imagen para el Diagnóstico e Higienista Bucodental).

\section{INSTRUMENTOS}

La evaluación de variables sociodemográficas se llevó a cabo mediante el empleode un cuestionario elaborado ad hoc, 
que consta de seis preguntas con respuesta cerrada de dos o más alternativas.

El Brief Emotional Intelligence Inventory for Senior Citizens (EQ-I-M20) (Baron y Parker, 2000) fue utilizada para la evaluación de la inteligencia emocional. Concretamente, se aplicó la adaptación para población española adulta de Pérez-Fuentes, Gázquez, Mercader y Molero (2014). Este inventario consta de 20 ítems con formato de respuesta tipo Likert de 4 puntos (de $1=$ Nunca me pasa a $4=$ Siempre me pasa), que se distribuyen a través de 5 factores: intrapersonal (hace referencia a la habilidad para manejar y ser consciente de las propias emociones; p.e.: me resulta fácil decirle a la gente cómo me siento), interpersonal (se refiere a la capacidad para controlar y manejar los sentimientos ajenos; p.e.: sé cómo se sienten las otras personas), manejo del estrés (en relación al control de la tensión; p.e.: me resulta difícil controlar mi ira), adaptabilidad (en cuanto a la habilidad para afrontar situaciones novedosas; p.e.: puedo resolver problemas de diferentes maneras) y estado de ánimo (referente a la actitud emocional; p.e.: me siento seguro de mí mismo). La consistencia interna del instrumento fue de .88 para el factor intrapersonal, .70 en interpersonal, .85 en el caso del manejo del estrés y de .80 y.86 en el caso de adaptabilidad y estado de ánimo, respectivamente.

Para la evaluación del engagement académico, se utilizó el Utrecht Work Engagement Scale (UWES; Schaufeli \& Bakker, 2003) en su adaptación al español para estudiantes (UWES-S; Valdez y Ron, $2011)$. Esta escala, formada por 17 ítems, se responde a través de una escala tipo Likert de 7 puntos (donde 0 es "nunca" y 6 "siempre"). Estos ítems se distribuyen a través de tres factores: vigor (que hace referencia a la energía para enfrentar los estudios; p.e.: en mi trabajo me siento lleno de energía), dedicación (relacionada con el entusiasmo con el que el estudiante se implica en el abordaje de sus estudios; p.e.: mi trabajo está lleno de significado y propósito) y absorción (referida a la concentración y bienestar que se da durante la realización de la actividad; p.e.: el tiempo vuela cuando estoy trabajando). La fiabilidad hallada en cada uno de estos factores fue de $.85, .89$ y .82 , respectivamente.

\section{PROCEDIMIENTO}

El estudio fue aprobado por el Comité de Bioética de la Universidad de Almería (Ref: UALBIO2017/011). Los participantes accedieron a formar parte del estudio de manera voluntaria, siendo informados del anonimato y confidencialidad de sus respuestas.

La implementación de los cuestionarios fue realizada a través de una plataforma web, donde, además de los cuestionarios seleccionados, se incluyeron preguntas control para detectar a posibles participantes que contestasen de manera aleatoria 0 incongruente. Las respuestas de dichos participantes fueron eliminadas tras el filtrado de los datos.

\section{ANÁLISIS DE DATOS}

El análisis estadístico se llevó a cabo mediante el programa SPSS versión 23.0. Los parámetros descriptivos fueron realizados a través de análisis de frecuencias y descriptivos. Por otro lado, con el fin de examinar las posibles diferencias estadísticamente significativas en los factores del engagement y en inteligencia emocional en función del tipo de la titulación cursada y el sexo de los participantes, se aplicó la prueba + de Student, junto al estadístico d de Cohen para conocer el tamaño del efecto.

Igualmente, se utilizó la prueba de correlación de Pearson para determinar la relación entre la edad, la inteligencia emocional y los factores del compromiso académico de los estudiantes evaluados.

\section{RESULTADOS}

\section{ENGAGEMENT E INTELIGENCIA EMOCIONAL EN FUNCIÓN DEL SEXO Y TITULACIÓN}

Los resultados obtenidos en las dimensiones del engagement y la inteligencia emocional en función del sexo de los participantes 
se muestran en la Tabla 1. Tal y como se observa, se encontraron diferencias estadísticamente significativas entre hombres y mujeres para la dimensión estado de ánimo $\left(t_{(189)}=2.18 ; p<.05 ; d=.30\right)$ e intrapersonal de la inteligencia emocional $\left(t_{(189)}=-3.48\right.$; $p<.01 ; d=.48)$. Estas diferencias señalan que las mujeres presentan una situación emocional general más favorable y una mejor comprensión de sus emociones y sentimientos que sus compañeros del sexo masculino.

Igualmente, se encontraron diferencias estadísticamente entre ambos sexos para el factor vigor del engagement $\left(t_{(189)}=-2.23\right.$; $p<.05 ; d=.31)$. Concretamente, fueron las mujeres del área de Ciencias de la Salud quienes mostraron afrontar con mayor fuerza sus estudios.
Por otro lado, se analizaron las diferencias en engagement académico e inteligencia emocional en función del tipo de titulación de Ciencias de la Salud cursada por los participantes. Sin embargo, no se encontraron diferencias significativas entre los estudiantes universitarios y los de Formación Profesional (Tabla 2).

\section{RELACIÓN ENTRE LA EDAD, EL ENGAGEMENT $Y$ LA INTELIGENCIA EMOCIONAL EN ESTUDIANTES DE CIENCIAS DE LA SALUD}

Los resultados de la correlación entre la edad y los factores del engagement y la inteligencia emocional muestran, en primer lugar, que la edad se relaciona de manera negativa con la dedicación ( $r=-.12 ; p=.040)$.

\begin{tabular}{|c|c|c|c|c|c|c|c|c|}
\hline \multicolumn{9}{|c|}{$\begin{array}{c}\text { Tabla } 1 \\
\text { Inteligencia emocional y engagement. Descriptivos y prueba † según sexo }\end{array}$} \\
\hline & & \multicolumn{4}{|c|}{ Sexo } & \multirow{3}{*}{$t$} & \multirow{3}{*}{$p$} & \multirow{3}{*}{ d } \\
\hline & & \multicolumn{2}{|c|}{ Hombre $(n=74)$} & \multicolumn{2}{|c|}{ Mujer ( $n=189)$} & & & \\
\hline & & Media & $D T$ & Media & $D T$ & & & \\
\hline \multirow{3}{*}{ Engagement } & Vigor & 3.71 & 0.79 & 3.96 & 0.81 & $-2.23^{*}$ & .26 & .31 \\
\hline & Dedicación & 3.91 & 0.81 & 4.07 & 0.81 & -1.45 & .14 & .20 \\
\hline & Absorción & 3.50 & 0.76 & 3.72 & 0.81 & -1.97 & .05 & .27 \\
\hline \multirow{5}{*}{$\begin{array}{l}\text { Inteligencia } \\
\text { Emocional }\end{array}$} & Intrapersonal & 2.43 & 0.67 & 2.78 & 0.73 & $-3.48^{* *}$ & .001 & .48 \\
\hline & Interpersonal & 3.01 & 0.55 & 3.11 & 0.51 & -1.38 & .16 & .19 \\
\hline & Manejo del estrés & 3.40 & 0.57 & 3.23 & 0.65 & 1.89 & .05 & .26 \\
\hline & Adaptabilidad & 3.03 & 0.51 & 2.95 & 0.57 & 0.95 & .34 & .13 \\
\hline & & 3.31 & 0.57 & 3.13 & 0.61 & $2.18^{\star}$ & .03 & .30 \\
\hline
\end{tabular}

\begin{tabular}{|c|c|c|c|c|c|c|c|c|}
\hline \multicolumn{9}{|c|}{$\begin{array}{c}\text { Tabla } 2 \\
\text { Inteligencia emocional y engagement. Descriptivos y prueba † según estudios cursados }\end{array}$} \\
\hline & & \multicolumn{4}{|c|}{ Estudios cursados } & \multirow{3}{*}{$t$} & \multirow{3}{*}{$p$} & \multirow{3}{*}{$d$} \\
\hline & & \multicolumn{2}{|c|}{ Universitarios $(n=86)$} & \multicolumn{2}{|c|}{$\begin{array}{l}\text { Formación Profesional } \\
\qquad(n=173)\end{array}$} & & & \\
\hline & & Media & $D T$ & Media & $D T$ & & & \\
\hline \multirow{3}{*}{ Engagement } & Vigor & 3.94 & 0.75 & 3.85 & 0.83 & 0.78 & .43 & .10 \\
\hline & Dedicación & 4.09 & 0.77 & 3.98 & 0.83 & 0.96 & .33 & .13 \\
\hline & Absorción & 3.63 & 0.78 & 3.66 & 0.81 & -0.30 & .76 & .04 \\
\hline \multirow{5}{*}{$\begin{array}{l}\text { Inteligencia } \\
\text { Emocional }\end{array}$} & Intrapersonal & 2.65 & 0.70 & 2.68 & 0.75 & -0.36 & .71 & .05 \\
\hline & Interpersonal & 3.14 & 0.48 & 3.06 & 0.55 & 1.16 & .24 & .15 \\
\hline & Manejo del estrés & 3.23 & 0.57 & 3.30 & 0.66 & -0.90 & .365 & .12 \\
\hline & Adaptabilidad & 2.95 & 0.53 & 2.98 & 0.57 & -0.37 & .70 & .05 \\
\hline & Estado de Ánimo & 3.12 & 0.57 & 3.20 & 0.63 & -1.08 & .31 & .14 \\
\hline
\end{tabular}


De manera que, conforme avanza la edad, los estudiantes presentan una menor implicación en sus estudios.

Por otro lado, se observa que el factor vigor del engagement correlaciona de forma significativamente positiva con el resto de factores del engagement académico: dedicación $(r=.85 ; p=<.001)$ y absorción $(r=.84 ; p=<.001)$. Y también se relaciona de forma positiva intrapersonal $(r=.16 ; p=.006)$, interpersonal $(r=.32 ; p=<.001)$, manejo del estrés $(r=.19 ; p=.002)$, adaptabilidad $(r=.32 ; p=<.001)$ y estado de ánimo $(r=.34$; $p=<.001)$ de la inteligencia emocional. Por lo que, mayores niveles de voluntad y esfuerzo en los estudios se relacionan con mayor involucración y concentración en estos. Además de estar asociado con un nivel más elevado de conocimiento y manejo de los sentimientos propios y ajenos, control ante situaciones estresantes, capacidad para responder de forma rápida ante los cambios inesperados y mejor estado anímico.

Igualmente, encontramos una relación igualmente positiva significativa entre el factor dedicación del engagement académico con la absorción $(r=.82 ; p=<.001)$ y los factores de la inteligencia emocional: intrapersonal $(r=.20 ; \quad p=.001)$, interpersonal $(r=-.29$; $p=<.001)$, manejo del estrés $(r=.14$; $p=.017)$, adaptabilidad $(r=.27 ; p=<.001)$ y estado de ánimo $(r=.34 ; p=<.001)$. Por lo que un mayor afán y entusiasmo en los estudios se relaciona con mayor centración en estos y niveles más altos de inteligencia emocional.
Por último, también se hallaron correlaciones positivas significativas entre el factor absorción y los factores de la inteligencia emocional intrapersonal $(r=.17$; $p=.004)$, interpersonal $(r=.25 ; p=<.001)$, adaptabilidad $(r=.30 ; p=<.001)$ y estado de ánimo $(r=.34 ; p=<.001)$, a excepción del factor manejo del estrés. De manera que, la entrega a los estudios se relaciona con niveles más elevados de conocimiento y control de emociones, capacidad de respuesta ante situaciones novedosas y un estado de ánimo más positivo. Pero no con una mejor respuesta ante situaciones que generan estrés.

\section{DISCUSIÓN}

El objetivo de esta investigación fue analizar la relación entre los componentes del engagement académico y las dimensiones de la inteligencia emocional según la edad, el sexo y la titulación, en una muestra de estudiantes de Ciencias de la Salud. Los resultados han mostrado que existen diferencias significativas en función del sexo en la dimensión vigor del engagement y en el factor interpersonal de la inteligencia emocional, siendo el grupo de las mujeres el que presenta puntuaciones medias mayores, afrontando con más resistencia la carrera académica y mostrando una mejor comprensión de los sentimientos y emociones respecto al grupo de hombres. Estos resultados van en consonancia con el trabajo de Salmela-Aro et al. (2017), donde es el sexo femenino el que muestra un mayor compromiso académico en comparación al

Tabla 3

Matriz de correlación entre las variables Edad, Inteligencia emocional y Engagement

\begin{tabular}{|c|c|c|c|c|c|c|c|c|}
\hline & Vigor & Dedicación & Absorción & Intrapersonal & Interpersonal & $\begin{array}{l}\text { Manejo del } \\
\text { estés }\end{array}$ & Adaptabilidad & $\begin{array}{l}\text { Estado de } \\
\text { ánimo }\end{array}$ \\
\hline Edad & -.23 & $-.12^{*}$ & -.04 & .08 & -.02 & .09 & -.03 & -.07 \\
\hline Vigor & & $.85^{\star \star *}$ & $.84^{\star \star \star}$ & $.16^{* \star}$ & $.32^{\star \star \star}$ & $.19^{* \star}$ & $.32^{\star \star *}$ & $.34^{\star * *}$ \\
\hline Dedicación & & & $.82^{\star \star \star}$ & $.20 * \star$ & $.29 * \star \star$ & $.14^{*}$ & $.27^{\star \star \star}$ & $.34^{\star * *}$ \\
\hline Absorción & & & & $.17^{* *}$ & $.25^{\star \star \star}$ & .11 & $.30 * \star *$ & $.27^{\star \star \star}$ \\
\hline Intrapersonal & & & & & $.43^{\star \star \star}$ & -.07 & $.35^{\star \star \star}$ & $.21^{\star \star \star}$ \\
\hline Interpersonal & & & & & & .05 & $.56^{\star \star \star}$ & $.32^{\star \star \star}$ \\
\hline Manejo del estrés & & & & & & & .06 & $.34^{\star \star \star}$ \\
\hline Adaptabilidad & & & & & & & & $.46^{\star \star \star}$ \\
\hline
\end{tabular}


sexo masculino. Sin embargo, en las dimensiones de la inteligencia emocional hallamos que los hombres son los que presentan mayores puntuaciones medias en el estado de ánimo, respecto a las alcanzadas por las mujeres. Al igual, que ocurre en el trabajo de Amizi et al. (2010) que es el grupo de los hombres el que presenta mayores puntuaciones en las dimensiones estado de ánimo, manejo del estrés y adaptabilidad.

Respecto al compromiso académico y la inteligencia emocional según la titulación, damos respuesta a la segunda hipótesis formulada, encontrando que no existen diferencias significativas entre el grupo de universitarios y el grupo de formación profesional. Estos resultados pueden deberse a la composición de la muestra, pues en otras investigaciones si han mostrado la existencia de diferencias significativas entre los estudiantes universitarios y los de formación profesional, en este caso en la variable intrapersonal de la inteligencia emocional (Martos et al., 2018).

A partir de los resultados obtenidos, en la correlación entre las dimensiones de la inteligencia emocional, los componentes del engagement y la edad respondemos a otra de las hipótesis planteadas, hallando entre la edad y la dedicación una relaciónsignificativa y negativa. Es decir, los alumnos con mayor edad suelen presentar una menor dedicación en los estudios, y los estudiantes de menor edad una mayor dedicación académica (Manzano, 2002). Del mismo modo, se ha encontrado una asociación positiva entre todas las dimensiones de la inteligencia emocional y los componentes del engagement, excepto entre la absorción y el manejo del estrés, donde no se ha observado una relación estadísticamente significativa. Resultados en línea con la investigación de Serrano y Andrea (2016), donde mostraron una asociación entre vigor y dedicación, y la atención y reparación emocional, en una muestra de adolescentes.

En cuanto a las limitaciones detectadas en el presente trabajo: el diseño transversal es una de ellas, pues sería conveniente realizar una investigación longitudinal, desde el comienzo de sus estudios hasta el final, para justificar la evolución y significación que se establece entre las variables de estudio. Otra limitación es el tamaño y composición de la muestra, dado el escaso número de participantes y la diversidad de titulaciones. Por tanto, para futuras investigaciones sería necesario homogenizar y ampliar la muestra, y corroborar si las diferencias en función del sexo en cuanto a los factores estudiados, son debidas a la muestra seleccionada. Asimismo, dada la escasez de trabajos realizados en estudiantes universitarios y de educación superior que analicen y contrasten ambas variables de estudio, encontramos otra limitación a la hora de respaldar y contrastar los resultados obtenidos. A esto cabría añadir, que la media de edad obtenida en la muestra es demasiado alta para ser estudiantes universitarios y de formación profesional.

En definitiva, los resultados revelan la asociación entre el compromiso académico y la inteligencia emocional en estudiantes de Ciencias de la Salud. Por tanto, este trabajo aporta resultados que destacan el importante papel que juegan las emociones en la toma de decisiones de los estudiantes y en el compromiso hacia los estudios, pues un buen manejo del estrés, estado de ánimo y adaptabilidad hará que los alumnos muestren una mayor dedicación y concentración a su labor. No dependiendo el engagement académico de la carrera profesional seleccionada. Pero, si se han encontrado diferencias según el sexo en la dimensión vigor. Además, este trabajo contribuye con datos acerca de una población específica, como son los estudiantes de Formación Profesional, de los cuales hay escasas investigaciones relacionadas con estas variables en la literatura científica.

Al mismo tiempo, destacar las implicaciones prácticas de los resultados alcanzados, principalmente para la creación y diseño de intervenciones, donde se tendrá que tener en cuenta las variables más destacadas, proporcionando así información a los docentes acerca de la importancia de la inteligencia emocional en el compromiso académico de los estudiantes de Ciencias de la Salud. Pues un control de las emociones mantendrá un mayor interés hacia los estudios en las distintas etapas educativas y por tanto el rendimiento no se verá afectado. 
- Conflicto de intereses.

Los autores declaran no tener ningún conflicto de intereses.

\section{REFERENCIAS}

Andrade, C.A., Nobrega, N., y Franco, M.G. (2016). Emotional intelligence and engagement in teachers of basic and secondary education of Madeira Island. Revista de Estudios e Investigación en Psicología y Educación, 3(2), 21-130. doi: 10.17979/reipe.2016.3.2.1810

Azimi, S., Asghar Nejad, A.A., Kharazi, M.J., y Khoei, N. (2010). Emotional intelligence of dental students and patient satisfaction. European Journal of Dental Education, 14(3), 129-132. doi: 10.1111/i.1600-0579.2009.00596.x

Bakker, A.B., Albrecht, S.L., y Leiter, M.P. (2011). Key questions regarding work engagement. European Journal of Work and Organizational Psychology, 20, 4-28. doi: 10.1080/1359432X. 2010.485352

Barnett, M.D., Melugin, P.R., y Hernández, J. (2018). Time perspective, intended academic engagement, and academic performance. Current Psychology, 1-7. doi: 10.1007/s12144-017-9771-9

Bar-On, R., y Parker, J.D.A (2000). The BarOn Emotional Quotient Inventory: youth version (EQ-i: YV). Technical Manual. MultiHealthSystems: Toronto, Canadá, 2000.

Carvalho, V.S., Guerrero, E., y Chambel. M.J. (2018). Emotional intelligence and health students' well-being: A twowave study with students of medicine, physiotherapy and nursing. Nurse Education Today, 63, 35-42. doi: 10.1016/i.nedt.2018.01.010

Casuso-Holgado, M.J., Cuesta-Vargas, A.I., Moreno-Morales, N., LabajosManzanares, M.T., Barón-López, F.J., y Vega-Cuesta, M. (2013). The association between academic engagement and achievement in health sciences students. BMC Medical Education, 13(1), 13-33. doi: 10.1 186/1472-6920-13-33

Collet, J., de Vugt, M.E., Schols, J.M.G.A., Engelen, G.J.J.A., Winkens, B., y Verhey, F.R.J. (2018). Well-being of nursing staff on specialized units for older patients with combined care needs. Journal of Psychiatric and Mental Health Nursing, 25, 108-118. doi: 10.1111/ipm.12445

Duran, A., Extremera, N., Rey, L., FernándezBerrocal, P., y Montalbán, F.M. (2006). Predicting academia burnout and engagement in educational settings: Assessing the incremental validity of perceived emotional intelligence beyond perceived stress and general self-efficacy. Psicothema, 18, 158-164.

Lisbona, A., Palaci, F., Salanova, M., y Frese, M. (2018). The effects of work engagement and self-efficacy on personal initiative and performance. Psicothema, 30(1), 89-96. doi: 10.7334/ psicothema2016.245

Maguire, R., Egan, A., Hyland, P., y Maguire, P. (2017). Engaging students emotionally: the role of emotional intelligence in predicting cognitive and affective engagement in higher education. Higher Education Research \& Development, 27(3-4), 197-214. doi: 10.1080/07294360.2016.1185396

Manzano, G. (2002). Burnout y engagement en un colectivo preprofesional estudiantes universitarios. Boletín de Psicología, 74, 79-102.

Martos, Á., Pérez-Fuentes, M.C., Molero, M.M., Gázquez, J.J., Simón, M.M., y Barragán, A.B. (2018). Burnout y engagement en estudiantes de Ciencias de la Salud. European Journal of Investigation in Health, Psychology and Education, 8(1), 23-36. doi: 10.30552/ ejihpe.v8i1.223

Merino, E., Hontangas, P.M., y Petrides, K.V. (2018). Career adaptability mediates the effect of trait emotional intelligence on academic engagement. Revista de Psicodidáctica, 23(2), 77-85. doi: 10.1016/i.psicoe.2017.10.002

Merino-Tejedor, E., Hontangas, P.M., y Boada-Grau, J. (2016). Career adaptability and its relation to selfregulation, career construction, and academic engagement among Spanish university students. Journal of Vocational 
Behavior, 93, 92-102. doi: 10.1016/i. jvb.2016.01.005

Morales, F. (2017). Relaciones entre afrontamiento del estrés cotidiano, autoconcepto, habilidades sociales e inteligencia emocional. European Journal of Education and Psychology, 10(2), 41 48. doi: 10.1016/i.ejeps.2017.04.001.

Nilforooshan, P., y Salimi, S. (2016). Career adaptability as a mediator between personality and career engagement. Journal of Vocational Behavior, 94, 1-10. doi: 10.1016/i.jvb.2016.02.010

Oriol, X., Amutio, A., Mendoza, M., Da Costa, S., y Miranda, R. (2016). Emotional creativity as predictor of intrinsic motivation and academic engagement in university students: the mediating role of positive emotions. Frontiers in Psychology, 7, 1-9. doi: 10.3389/fpsyg.2016.01243 Oriol-Granado, X., Mendoza-Lira, M., Covarrubias-Apablaza, C.G., y MolinaLópez, V.M. (2017). Positive emotions, autonomy support and academic performance of university students: the mediating role of academic engagement and self-efficacy. Revista de Psicodidáctica, 22(1), 45-53. doi: 10.1016/S1136-1034(17)30043-6.

Perera, H.N., y DiGiacomo, M. (2015). The role of trait emotional intelligence in academic performance during the university transition: An integrative model of mediation via social support, coping, and adjustment. Personality and Individual Differences, 83, 208-213. doi: 10.1016/i.paid.2015.04.001

Pérez-Fuentes, M.C., Molero, M.M., Gázquez, J.J., y Oropesa, N.F. (2018). The Role of emotional intelligence in engagement in nurses. International Journal of Environmental Research and Public Health, 15, 1915. doi: 10.3390/ iierph 15091915

Pérez-Fuentes, M.C., Molero, M.M., Gázquez, J.J., y Simón, M.M. (2019). Analysis of burnout predictors in nursing: risk and protective psychological factors. The European Journal of Psychology Applied to Legal Context, $17(1), 33-40$. doi: 10.5093/ejpalc2018a13

Pérez-Fuentes, M.C., Gázquez, J.J., Mercader, I., y Molero, M.M. (2014). Brief emotional intelligence inventory for senior citizens (EQ-I-M20). Psicothema, 26, 524-530.

Salanova, M., Bresó, E., y Schaufeli, W.B. (2005). Hacia un modelo espiral de las creencias de eficacia en el estudio del burnout y del engagement. Ansiedad y Estrés, 17 (2-3), 215-231.

Salmela-Aro, K., y Read, S. (2017). Study engagement and burnout profiles among Finnish higher education students. Burnout Research, 7, 21-28. doi: 10.1016/i. burn.2017.11.001

Sánchez-García, M.A., Lucas-Molina, B., Fonseca-Pedrero, E., Pérez-Albéniz, A., y Paino, M. (2018). Emotional and behavioral difficulties in adolescence: Relationship with emotional well-being, affect, and academic performance. Anales de Psicología, 34(3), 482-489. doi: 10.6018 /analesps.34.3.296631

Schaufeli, W.B., Salanova, M., GonzálezRomá, V., y Bakker, A. (2002). The measurement of burnout and engagement: A confirmatory factor analytic approach. Journal of Happiness Studies, 3, 71-92. doi: 10.1023/A:1015630930326

Schaufeli, W.B., y Bakker, A. (2003). UWES, Utrecht Work Engagement Scale. Utrecht University: Utrecht, The Netherlands.

Serrano, C., y Andreu, Y. (2016). Perceived emotional intelligence, subjective wellbeing, perceived stress, engagement and academic achievement of adolescents. Revista de Psicodidáctica, $27(2), \quad 357-374$. doi: $\underline{10.1387 /}$ RevPsicodidact. 14887

Urquiio, I., Extremera, N., y Villa, A. (2016). Emotional intelligence, life satisfaction, and psychological wellbeing in graduates: the mediating effect of perceived stress. Applied Research in Quality of Life, $17(4), 1241-1252$. doi: 10.1007/s $11482-015-9432-9$.

Valdez, H., y Ron, C. (201 1). UWES. Utrecht Work Engagement Scale.Escala Utrecht 
de Engagement en el Trabajo. Módulo de Atención Integral de la Comisaría General de Prevención y Reinserción Social del Estado de Jalisco: Ciudad de México, México.

Wach, F.S., Karbach, J., Ruffing, S., Brünken, R., y Spinath, F.M. (2016). University students' satisfaction with their academic studies: personality and motivation matter. Frontiers in Psychology, 7(55), 1 - 12. doi: 10.3389/fpsyg.2016.00055. Wersebe, H., Lieb, R., Meyer, A.H., Hofer, P., y Gloster, A.T. (2018). The link between stress, well-being, and psychological flexibility during an Acceptance and Commitment Therapy self-help intervention. International Journal of Clinical and Health Psychology, 18(1), 6068. doi: 10.1016/i.ijchp.2017.09.002

Zhang, Y., Qin, X., y Ren, P. (2018). Adolescents' academic engagement mediates the association between Internet addiction and academic achievement: The moderating effect of classroom achievement norm. Computers in Human Behavior, 89, 299-307. doi: 10.1016/i. chb.2018.08.018 